\title{
Algebraic Cryptanalysis of 58-Round SHA-1
}

\author{
Makoto Sugita ${ }^{1}$, Mitsuru Kawazoe ${ }^{2}$, Ludovic Perret ${ }^{3}$, and Hideki Imai ${ }^{4}$ \\ ${ }^{1}$ IT Security Center, Information-technology Promotion Agency, Japan \\ 2-28-8 Honkomagome, Bunkyo-ku Tokyo, 113-6591, Japan \\ m-sugita@ipa.go.jp \\ ${ }^{2}$ Faculty of Liberal Arts and Sciences \\ Osaka Prefecture University \\ 1-1 Gakuen-cho Naka-ku Sakai Osaka 599-8531 Japan \\ kawazoe@las.osakafu-u.ac.jp \\ ${ }^{3}$ SPIRAL/SALSA \\ Site Passy-Kennedy \\ LIP6 - Paris 6 University \\ 104 avenue du Président Kennedy \\ 75016 Paris France \\ ludovic.perret@lip6.fr \\ ${ }^{4}$ National Institute of Advanced Industrial Science and Technology (AIST) \\ Akihabara Dai Bldg., 1-18-13 Sotokanda, Chiyoda-ku, Tokyo 101-0021, Japan \\ Department of Electrical, Electronic and Communication Engineering \\ Faculty of Science and Engineering, Chuo University \\ 1-13-27 Kasuga Bunkyo-ku, Tokyo 112-8551 Japan \\ h-imai@aist.go.jp
}

\begin{abstract}
In 2004, a new attack against SHA-1 has been proposed by a team leaded by Wang 15 . The aim of this article 1 is to sophisticate and improve Wang's attack by using algebraic techniques. We introduce new notions, namely semi-neutral bit and adjuster and propose then an improved message modification technique based on algebraic techniques. In the case of the 58-round SHA-1, the experimental complexity of our improved attack is $2^{31}$ SHA- 1 computations, whereas Wang's method needs $2^{34}$ SHA-1 computations. We have found many new collisions for the 58-round SHA-1. We also study the complexity of our attack for the full SHA-1.
\end{abstract}

Keywords: SHA-1, Gröbner basis, differential attack.

\section{Introduction}

Conceptually, we can split Wang's attack in four different steps.

Step 1. Choose a suitable $80 \times 32$-bit vector $\Gamma$.

Step 2. Choose a differential characteristic.

${ }^{1}$ A part of this work has been done when the third author was invited at Research Center for Information Security (RCIS) at Tokyo (Japan). 
Step 3. Find a set of sufficient conditions on a message $m$ and the chaining variables which guarantees with high probability that the message pair $(m, m+\Gamma)$ follows the differential characteristic. This implies that the two messages do collide.

Step 4. Choose a message $m$ randomly and modify it until all sufficient conditions hold.

Using this method, Wang's team succeeded in finding collisions on the most popular hash functions, namely MD4, MD5, RIPEMD, SHA-0 and 58-round SHA-1 1117 15. The attack is conceptually simple, but its implementation turns out to be very laborious in practice. To fill this gap between theory and practice, several teams decided to compensate their lack of intuition by the power of a computer, that is to say they tried to automatize the different steps of the attack.

For instance, coding theory can be used for finding a suitable difference in Step 1 [8] of the attack. Recently, De Cannière and Rechberger [3] presented an algorithm allowing to find optimal differential characteristics in Step 2. The third step is tightly coupled with the previous one; most sufficient conditions follow from the choice of the differential characteristic.

We use algebraic techniques for actually finding collisions on 58-round SHA-1. In this case, the complexity of our method for finding a collision is equivalent to $2^{31}$ SHA-1 computations (experimentally), whereas Wang's method needs $2^{34}$ SHA-1 computations. As a proof of concept, we have found many new collisions for 58-round SHA-1, which have never been reported so far. We also apply our method for the case of the full SHA-1, and study the complexity of our approach.

The key idea is to describe the message modification technique into an algebraic framework. This is done by viewing the set of sufficient conditions as a non-linear system of Boolean equations. We hope that this will be a first step towards the use of algebraic tools (such as Gröbner bases) in the cryptanalysis of hash functions.

We will focus our attention on the last step of Wang's et al attack [15]. Namely, find a message satisfying a set of sufficient conditions depending on a disturbance vector and a differential path. This message can be then use to produce a collision. We shall call conventional message modification the process [15] permitting to construct such a suitable message. Here, we will present an improved message modification technique. To do so, we introduce the concepts semi-neutral bits and adjusters.

This paper is organized as follows. In Section 2, we give a description of SHA-1. Along the way, we introduce the notations and definitions that will be used throughout this paper. In Section 3, we describe our improved message modification technique. We explain how to use Gaussian elimination to construct a set controlled relations from the sufficient conditions. We also introduce a new notion, that we called semi-neutral bit, and describe then our improved message modification. We also give an algebraic descriptions of our improved message modification. This permits to give an interesting connection between the cryptanalysis of hash functions and the use of Gröbner bases. In Section 4 . 
we present the details of our method on 58-round SHA-1. In the appendix, we provide the details for the full SHA-1.

\section{Preliminaries}

\subsection{Description of SHA-1}

The hash function SHA-1 generates a 160-bit hash value (or digest) from a message of length less than $2^{64}$ bits. The input message is padded and then processed in 512-bit message blocks through the Merkle/Damgard iterative structure.

A 80-step compression function is then applied to each of these 512-bit message blocks. It has two types of inputs: a chaining input of 160 bits and a message input of 512 bits. The initial chaining value (called IV) is a set of fixed constants, and the result of the last call to the compression function is the hash of the message.

In SHA-1, the message expansion is defined as follows: each 512-bit block of the padded message is divided into a $16 \times 32$-bit word $\left(m_{0}, m_{1}, \ldots, m_{15}\right)$, and then expanded according to the following linear relation :

$$
m_{i} \leftarrow\left(m_{i-3} \oplus m_{i-8} \oplus m_{i-14} \oplus m_{i-16}\right) \lll 1, \text { for all } i, 16 \leq i \leq 79,
$$

$x \lll n$, denoting the $n$-bit left rotation of a 32 -bit word $x$. The compression function is defined for all $i, 1, \leq i \leq 80$ as follows:

$$
\begin{aligned}
a_{i} & \leftarrow\left(a_{i-1} \lll 5\right)+f_{i}\left(b_{i-1}, c_{i-1}, d_{i-1}\right)+e_{i-1}+m_{i-1}+k_{i} \\
b_{i} & \leftarrow a_{i-1} \\
c_{i} & \leftarrow b_{i-1} \lll 30 \\
d_{i} & \leftarrow c_{i-1} \\
e_{i} & \leftarrow d_{i-1}
\end{aligned}
$$

The initial chaining value $\mathrm{IV}=\left(a_{0}, b_{0}, c_{0}, d_{0}, e_{0}\right)$ being equal to:

(0x67452301, 0xefcdab89, 0x98badcfe, 0x10325476, 0xc3d2e1f0).

Note that we express as usual 32-bit words as hexadecimal numbers.

For $n, 58 \leq n \leq 80$, we call $n$-round SHA-1, the restriction of SHA-1 to the first $n$ rounds. The Boolean function $f_{i}$ and constant $k_{i}$ employed at each step are defined as in Table 1

Table 1. Definition of $f_{i}$ and $k_{i}$ w.r.t. the step

\begin{tabular}{|l|l|l|}
\hline Step & Boolean function $f_{i}$ & Constant $k_{i}$ \\
\hline $1-20$ & IF: $(x \wedge y) \vee(\neg x \wedge z)$ & 0x5a827999 \\
$21-40$ & XOR: $x \oplus y \oplus z$ & 0x6ed6eba 1 \\
$41-60$ & MAJ: $(x \wedge y) \vee(x \wedge z) \vee(y \wedge z)$ & $0 x 8$ fabbcdc \\
$61-80$ & XOR: $x \oplus y \oplus z$ & $0 x c a 62 c 1 d 6$ \\
\hline
\end{tabular}




\subsection{Definition and Notation}

We will identify the ring $\mathbb{Z} / 2^{32} \mathbb{Z}$ with $\left\{0,1,2, \ldots, 2^{32}-1\right\}$. If we ignore carry effects in the arithmetic of $\mathbb{Z} / 2^{32} \mathbb{Z}$, we can identify the ring $\mathbb{Z} / 2^{32} \mathbb{Z}$ with the vector space $\mathbb{F}_{2}^{32}$ by using the canonical bijective mapping $\varrho$ :

$$
\varrho: x_{31} 2^{31}+x_{30} 2^{30}+\cdots+x_{1} 2^{1}+x_{0} 2^{0} \in \mathbb{Z} / 2^{32} \mathbb{Z} \longmapsto\left(x_{31}, x_{30}, \ldots, x_{0}\right) \in \mathbb{F}_{2}^{32} .
$$

Here and in the rest of the paper, we try to find a collision between two messages $m=\left(m_{0}, m_{1}, \ldots, m_{79}\right)$ and $m^{\prime}=\left(m_{0}^{\prime}, m_{1}^{\prime}, \ldots, m_{79}^{\prime}\right)$ of $\left(\mathbb{F}_{2}^{32}\right)^{80}$. The corresponding chaining variables will be denoted by $a_{i}, b_{i}, c_{i}, d_{i}, e_{i}$ and $a_{i}^{\prime}, b_{i}^{\prime}, c_{i}^{\prime}, d_{i}^{\prime}, e_{i}^{\prime}$ respectively. For each $m_{i}=\left(m_{i, 31}, m_{i, 30}, \ldots, m_{i, 0}\right)$ and $m_{i}^{\prime}=\left(m_{i, 31}^{\prime}, m_{i, 30}^{\prime}, \ldots, m_{i, 0}^{\prime}\right)$, we define:

$$
\begin{aligned}
\Delta m_{i, j} & =m_{i, j} \oplus m_{i, j}^{\prime} \in \mathbb{F}_{2}, \\
\Delta m_{i} & =m_{i} \oplus m_{i}^{\prime} \in \mathbb{F}_{2}^{32}, \\
\delta m_{i} & =\varrho^{-1}\left(m_{i}\right)-\varrho^{-1}\left(m_{i}^{\prime}\right) \in \mathbb{Z} / 2^{32} \mathbb{Z} .
\end{aligned}
$$

Moreover, we set :

$\Delta^{+} m_{i, j}=\left\{\begin{array}{ll}1 & \text { if }\left(m_{i, j}, m_{i, j}^{\prime}\right)=(0,1) \\ 0 & \text { otherwise }\end{array} \quad \Delta^{-} m_{i, j}= \begin{cases}1 & \text { if }\left(m_{i, j}, m_{i, j}^{\prime}\right)=(1,0) \\ 0 & \text { otherwise }\end{cases}\right.$ and

$$
\Delta^{+} m_{i}=\left(\Delta^{+} m_{i, 31}, \ldots, \Delta^{+} m_{i, 1}, \Delta^{+} m_{i, 0}\right), \quad \Delta^{-} m_{i}=\left(\Delta^{-} m_{i, 31}, \ldots, \Delta^{-} m_{i, 1}, \Delta^{-} m_{i, 0}\right) .
$$

Note that $\Delta m_{i}=\Delta^{+} m_{i} \oplus \Delta^{-} m_{i}^{\prime} \in \mathbb{F}_{2}^{32}$. Similarly, we define $\Delta, \Delta^{+}, \Delta^{-}, \delta$ for the chaining variables $b_{i}, c_{i}, d_{i}$ and $e_{i}$ (resp. $b_{i}^{\prime}, c_{i}^{\prime}, d_{i}^{\prime}$ and $e_{i}^{\prime}$ ). Using the above definition, a differential characteristic and a differential are defined as follows.

Definition 1. We call differential characteristic the sequence:

$$
\left(\Delta m_{i}, \Delta a_{i}, \Delta b_{i}, \Delta c_{i}, \Delta d_{i}, \Delta e_{i}\right)_{0 \leq i \leq 79},
$$

and differential:

$$
\left(\Delta^{+} m_{i}, \Delta^{-} m_{i}, \Delta^{+} a_{i}, \Delta^{-} a_{i}, \ldots, \Delta^{+} e_{i}, \Delta^{-} e_{i}\right)_{0 \leq i \leq 79} .
$$

\section{An Improved Message Modification Technique}

Here, we will consider a $n$-round SHA-1, with $n, 58 \leq n \leq 80$. We will focus our attention on the last step of Wang's attack. Thus, we will suppose that a disturbance vector is fixed, as well as a suitable differential. We can then determine sufficient conditions on the messages permitting to produce collisions. Remark that sufficient conditions depend on the choice of a disturbance vector and its differential. 


\subsection{How to Calculate Sufficient Conditions on the $a_{i}$ ?}

In this step, we only consider expanded messages by ignoring relations arising from the message expansion. We compute the sufficient conditions on chaining variables by adjusting $b_{i}, c_{i}$ and $d_{i}$ such that for all $i, 0 \leq i \leq n-1$ :

$$
\delta f_{i}\left(b_{i}, c_{i}, d_{i}\right)=\delta a_{i+1}-\left(\delta a_{i} \lll 5\right)-\delta e_{i}-\delta m_{i} .
$$

In this calculation, we must adjust carry effects by "hand". It is indeed difficult to calculate this full-automatically. In Table 2 and Table 6 , we present the sufficient conditions that we have obtained on the chaining variables for 58-round and the full SHA-1, respectively. Note that sufficient conditions on the messages are also quoted in this table.

\subsection{Gaussian Elimination and Controlled Relations}

To calculate sufficient conditions on the $\left\{m_{i, j}\right\}_{0 \leq i \leq n-1}^{0 \leq j \leq 31}$, we take into account that $\Delta^{+} m_{i, j}=1$ implies $m_{i, j}=0$ and $\Delta^{-} m_{i, j}=0$ implies $m_{i, j}=1$. We also consider the relations derived from the key expansion:

$$
m_{i, j} \leftarrow\left(m_{i-3, j} \oplus m_{i-8, j} \oplus m_{i-14, j} \oplus m_{i-16, j}\right) \lll 1 \text {, for all } i, 16 \leq i \leq n-1 .
$$

We shall call controlled relations a particular set of $\mathbb{F}_{2}$-linear equations on the $m_{i, j}$ on one hand, and on the chaining variables $a_{i, j}$ on the other. For the $m_{i, j}$, we consider the relations obtained by performing a Gaussian elimination on the linear equations defined by the key expansion, and the equations derived from the sufficient conditions. To perform this Gaussian elimination, we have considered the following order on the $m_{i, j}$ :

$$
m_{i^{\prime}, j^{\prime}}<m_{i, j} \text {, if } i^{\prime}<i \text { or } i^{\prime}=i \text { and } j^{\prime}<j \text {. }
$$

For $n=58$, we obtain for instance the following controlled relations :

$$
\begin{aligned}
& m_{15,31}=1, m_{15,30}=1, m_{15,29}=0, m_{15,28}+m_{10,28}+m_{8,29}+m_{7,29}+m_{4,28}+m_{2,28}=1, \\
& m_{15,27}+m_{14,25}+m_{12,28}+m_{12,26}+m_{10,28}+m_{9,27}+m_{9,25}+m_{8,29}+m_{8,28}+m_{7,28}+ \\
& m_{7,27}+m_{6,26}+m_{5,28}+m_{4,26}+m_{3,25}+m_{2,28}+m_{1,25}+m_{0,28}=1, m_{15,26}+m_{10,28}+ \\
& m_{10,26}+m_{8,28}+m_{8,27}+m_{7,27}+m_{6,29}+m_{5,27}+m_{4,26}+m_{2,27}+m_{2,26}+m_{0,27}=1, \\
& m_{15,25}+m_{11,28}+m_{10,27}+m_{10,25}+m_{9,28}+m_{8,27}+m_{8,26}+m_{7,26}+m_{6,29}+m_{6,28}+ \\
& m_{5,26}+m_{4,25}+m_{3,28}+m_{2,28}+m_{2,26}+m_{2,25}+m_{1,28}+m_{0,28}+m_{0,26}=0, m_{15,24}+ \\
& m_{12,28}+m_{11,27}+m_{10,26}+m_{10,24}+m_{9,28}+m_{9,27}+m_{8,29}+m_{8,26}+m_{8,25}+m_{7,25}+ \\
& m_{6,29}+m_{6,28}+m_{6,27}+m_{5,25}+m_{4,28}+m_{4,24}+m_{3,28}+m_{3,27}+m_{2,27}+m_{2,25}+m_{2,24}+ \\
& m_{1,28}+m_{1,27}+m_{0,27}+m_{0,25}=1, m_{15,23}+m_{12,28}+m_{12,27}+m_{11,26}+m_{10,25}+m_{10,23}+ \\
& m_{9,27}+m_{9,26}+m_{8,28}+m_{8,25}+m_{8,24}+m_{7,29}+m_{7,24}+m_{6,28}+m_{6,27}+m_{6,26}+m_{5,24}+ \\
& m_{4,27}+m_{4,23}+m_{3,27}+m_{3,26}+m_{2,26}+m_{2,24}+m_{2,23}+m_{1,27}+m_{1,26}+m_{0,26}+m_{0,24}=1, \\
& m_{15,22}+m_{14,25}+m_{12,28}+m_{12,27}+m_{11,25}+m_{10,27}+m_{10,24}+m_{10,22}+m_{9,28}+m_{9,27}+ \\
& m_{9,26}+m_{8,27}+m_{8,24}+m_{8,23}+m_{7,28}+m_{7,27}+m_{7,23}+m_{6,27}+m_{6,25}+m_{5,23}+m_{4,28}+ \\
& m_{4,27}+m_{4,22}+m_{3,26}+m_{2,28}+m_{2,27}+m_{2,25}+m_{2,23}+m_{2,22}+m_{1,26}+m_{0,25}+m_{0,23}=0, \\
& \ldots, m_{5,0}+m_{3,0}+m_{1,31}=1, m_{4,31}=0, m_{4,30}=0, m_{4,29}=0, m_{4,6}=0, m_{4,1}=1,
\end{aligned}
$$


$m_{3,30}=1, m_{3,29}=0, m_{3,6}=1, m_{2,31}=0, m_{2,30}=1, m_{2,29}=0, m_{2,6}=1, m_{2,1}=1$, $m_{2,0}=1, m_{1,30}=0, m_{1,29}=1, m_{1,5}=0, m_{1,4}=1, m_{1,1}=1, m_{0,31}=0, m_{0,30}=0$, $m_{0,29}=0$.

The controlled relations also include a subset of the sufficient conditions on the chaining variables. Precisely, we will only consider the conditions involving $a_{i, j}$, with $i \leq R$. The bound $R$ is a positive integer that will be defined later. We will call uncontrolled relations, the sufficient conditions which are not a controlled relation.

We define now the notions of semi-neutral bit, control bit and adjuster. The concept of semi-neutral bit is closely related to Biham and Chen's "neutral bit" 2] and Klima's "tunnels" 22]. Namely, if the effect of flipping a bit corresponding to a chaining variable can be "easily" eliminated (i.e. such that all conditions previously satisfied can be satisfied by modifying few bits), then we shall call this bit a semi-neutral bit. Thus, the effect of changing a semi-neutral bit can be eliminated by controlling a little number of bits. We shall call these particular bits adjusters. Note that the choice of semi-neutral bits and adjusters is not unique. Thus, we have to choose it heuristically.

We emphasize that each $m_{i, j}$ can be viewed as a polynomial on the $a_{k, \ell}$ 's, with $k \leq i+1$. Indeed, each $m_{i, j}$ can be viewed as a Boolean function on the $a_{k, \ell}$ 's, with $k \leq i+1$, by the definition of SHA-1. Note that when we view $m_{i, j}$ as a Boolean function, we do not approximate (based on approximating MAJ by XOR, ignoring carry effect, etc.), but consider it as exact polynomial on the $a_{k, \ell}$. Control bits are determined for each controlled relation. Control bits are chosen among the $a_{k, \ell}$ which appear as a leading term or a term 'near' leading term in $m_{i, j}$, where $m_{i, j}$ is considered as a Boolean function on the $a_{k, \ell}$. The notion of leading term being related to a term ordering, we mention that we have considered here the following order on the $a_{k, \ell}$ :

$$
a_{k, \ell}<a_{k^{\prime}, \ell^{\prime}} \text { if } k^{\prime}<k \text { or } k^{\prime}=k \text { and } \ell^{\prime}<\ell .
$$

\subsection{Conventional/Advanced Message Modification Techniques}

The last step of Wang's attack consists of randomly choosing a message and modify some of its bits until all sufficient conditions are satisfied. To our knowledge, this technique has been described for the first time in [1819]. We shall call this method conventional message modification technique.

Here, we introduce an improved message modification. The conventional message modification will be used to obtain a "pre-collision", i.e. a collision from the first round to a given round $R$. This bound $R$ will depend on the number $n$ of rounds considered. We take $R=23$ in the case of 58-round SHA-1 and $R=26$ for the full SHA-1. The improved message modification will then allow to extend the pre-collision into a real collision on $n$-round SHA-1.

We would like to emphasize that our procedure will modify the chaining variable and not the message. Since $\operatorname{IV}=\left(a_{0}, b_{0}, c_{0}, d_{0}, e_{0}\right)$ is fixed, it is clear that 
SHA-1 induces a bijection between $\left(m_{0}, m_{1}, \ldots, m_{15}\right)$ and $\left(a_{1}, a_{2}, \ldots, a_{16}\right)$. This implies that a modification on the $a_{i, j}$ can be mapped into a modification on the $m_{i, j}$.

Using our new terminology, we describe the conventional message modification. For this, we use a list of controlled relations $C_{R}$, and a list of control bits $C_{B}$

\section{Algorithm 1. Conventional Message Modification CMM}

Input : A positive integer $R$, a list $C_{R}$ of controlled relations, and a list $C_{B}$ of control bits

Output : $\mathbf{a}=\left(a_{1}, a_{2}, \ldots, a_{16}\right) \in\left(\mathbb{F}_{2}^{32}\right)^{16}$ satisfying all the controlled relations Randomly choose $\left(a_{1}, a_{2}, \ldots, a_{16}\right) \in\left(\mathbb{F}_{2}^{32}\right)^{16}$

$\mathbf{a} \leftarrow\left(a_{1}, a_{2}, \ldots, a_{16}\right)$

While all the controlled relations $C_{B}$ are not satisfied do

Perform an exhaustive search on the bits $a_{i, j} \in C_{B}$

If all the relations $C_{B}$ are satisfied then return the updaded $\mathbf{a}$

Else $\mathbf{a} \leftarrow$ Randomly choose $\left(a_{1}, a_{2}, \ldots, a_{16}\right) \in\left(\mathbb{F}_{2}^{32}\right)^{16}$

The CMM algorithm permits then to find a collision on $R$-round SHA-1. Using semi-neutral bits and adjusters, we present an improved algorithm permitting to find a collision on a $n$-round SHA-1 (with $n>R$ ). The new procedure is as follows.

\section{Algorithm 2. Improved Message Modification IMM}

Input : Positive integers $n, R$, two lists $(S N B, A d)$ of semi-neutral bits and adjusters, a list $C_{R}$ of controlled relations, a list $C_{B}$ of control bits, and a list $S_{C}$ of sufficient conditions

Output : $\mathbf{a}=\left(a_{1}, a_{2}, \ldots, a_{16}\right) \in\left(\mathbb{F}_{2}^{32}\right)^{16}$ satisfying all the sufficient conditions $\mathbf{a}=\left(a_{1}, a_{2}, \ldots, a_{16}\right) \leftarrow C M M\left(R, C_{R}, C_{B}\right)$

While all the sufficient conditions of $S_{C}$ are not satisfied do

Adjust the $a_{i, j}$ of a corresponding to a semi-neutral bit of $S N B$ or an adjuster of $A d$

\section{EndWhile}

\section{Return a}

Remark 1. We mention that a different version of the CMM and IMM algorithms can be found in 910. These versions could be more suitable for those wishing to actually implement these two algorithms.

We now analyze the complexity of the IMM algorithm for finding a collision on 58-round SHA-1. In this case, we choose $R=23$, i.e. the CMM algorithm will be used to find a collision on 23-round SHA-1. It will remain 5 uncontrolled relations in rounds $17-23$. Therefore, the CMM algorithm needs at most $2^{5}$ 
iterations for returning a collision on 23-round SHA-1. There are 29 remaining conditions from rounds $23-58$. To adjust these 29 conditions, we use 21 semineutral bits and 16 adjusters. Experimentally, the total complexity is improved to $2^{31}$ SHA-1 computation - with our latest implementation - whereas Wang's method needs theoretically $2^{34}$ SHA-1 computations. Note that the cost of the IMM algorithm is dominated by the exhaustive search among 21 semi-neutral bits, which means that we could neglect the cost of the CMM algorithm.

As a proof of concept, we give here a new collision on 58-round SHA-1.

$$
\begin{aligned}
m= & 0 x 1 e a d 6636319 f e 59 e 4 e a 7 d d c b c 79616420 a d 9523 a f 98 f 28 d b 0 a d 135 d 0 e 4 d 62 a e c \\
& 6 c 2 d a 52 c 3 c 7160 b 606 e c 74 b 2 b 02 d 545 e b d d 9 e 4663 f 1563194 f 497592 d d 1506 f 9 \\
m^{\prime}= & 0 x 3 e a d 6636519 f e 5 a c 2 e a 7 d d 88 e 7961602 e a d 95278998 f 28 d 98 a d 135 d 1 e 4 d 62 a c c \\
& 6 c 2 d a 52 f 7 c 7160 e 446 e c 74 f 2502 d 540 c 1 d d 9 e 466 b f 1563596 f 497593 f d 150699
\end{aligned}
$$

\subsection{An Algebraic Description of the Improved Message Modification}

We present here an algebraic description of the IMM and CMM algorithms which could be useful for further improvements. For this, we remark that the CMM algorithm is equivalent to the solving of a polynomial system of equations via controlled relations with control bits as unknown variables. Similarly, the whileloop of the IMM algorithm is equivalent to the solving of an algebraic system of equations via sufficient conditions with semi-neutral bits and adjusters as unknown variables.

In other words, let $\mathbf{X}=\left\{X_{i, j}\right\}_{\substack{0 \leq j \leq i \leq n \\ 1 \leq i \leq n}}$ and let $\mathbb{F}_{2}[\mathbf{X}]$ be the polynomial ring over $\mathbb{F}_{2}$ whose variables are $\mathbf{X}$. Remark that sufficient conditions can be considered as polynomial equations via Boolean functions. Thus, they can be expressed as algebraic polynomials on the $a_{i, j}$. Therefore - by replacing each $a_{i, j}$ by the variable $X_{i, j}$ - we can associate a set of polynomials on $\mathbb{F}_{2}[\mathbf{X}]$ to the set of sufficient conditions. With an obvious notation, we shall call controlled polynomial (resp. uncontrolled polynomial) the polynomial associated to a controlled relation (resp. uncontrolled relation).

Let $J$ be an ideal in $\mathbb{F}_{2}[\mathbf{X}]$ generated by $\left\{X_{i, j}^{2}+X_{i, j}\right\}_{1 \leq i \leq n}^{0 \leq j \leq 31}$, i.e. $J=\left\langle X_{i, j}^{2}+\right.$ $\left.X_{i, j}\right\rangle_{\substack{0 \leq j \leq 31 \\ 1 \leq i \leq n}}$. Let then $B_{n}$ be a quotient ring $\mathbb{F}_{2}[\mathbf{X}] / J$. Note that $B_{n}$ represents the set of all Boolean functions on the variables $X_{i, j}$.

Let $\mathbf{f}=\left(f_{1}, f_{2}, \ldots\right)$ be the set of controlled polynomials. Note that all controlled polynomials of $\mathbf{f}$ are in the subring $\mathbb{F}_{2}\left[\left\{X_{i, j}\right\}_{1 \leq i \leq R}^{0 \leq j \leq 31}\right]$, where $R$ is determined by $n$ (for instance, $R=23$ when $n=58$ and $R=26$ when $n=80$ ).

For a randomly taken $\mathbf{a}=\left(a_{1}, a_{2}, \ldots, a_{16}\right) \in\left(\mathbb{F}_{2}^{32}\right)^{16}$, let $C_{R}(\mathbf{a})$ be the system obtain from the sufficient conditions by replacing each variables $X_{i, j}$ - not corresponding to a control bit - by $a_{i, j}$. Similarly, let $S_{C}(\mathbf{a})$ be the system obtain from the sufficient conditions by replacing each variables $X_{i, j}$ - not corresponding to a semi-neutral bit or adjuster - by $a_{i, j}$. In this setting, the CMM and IMM algorithms can roughly be described as follows: 
Algorithm 3. Algebraic Message Modification

Randomly choose $\left(a_{1}, a_{2}, \ldots, a_{16}\right) \in\left(\mathbb{F}_{2}^{32}\right)^{16}$

$\mathbf{a} \leftarrow\left(a_{1}, a_{2}, \ldots, a_{16}\right)$

Solve the algebraic system of equations $C_{R}(\mathbf{a})$ : The solutions correspond to affectations of control bits verifying all controlled polynomials Solve the algebraic system of equations $S_{C}(\mathbf{a})$ : The solutions correspond to affectations of semi-neutral bits and adjusters verifying all uncontrolled polynomials

Update a according to the solutions of the two previous systems Return a

Relation Between Message Modification and Decoding of Error- Correcting Codes. Let $S$ be the set of all points in $F=\left(\mathbb{F}_{2}^{32}\right)^{16}$ satisfying advanced sufficient conditions on $\left\{a_{i, j}\right\}$. Note that $S$ is a non-linear subset of $F$ because there are non-linear conditions. Then, for a given $\mathbf{a} \in F$ which is not necessarily contained in $S$, to find an element in $S$ by modifying a is analogous to a decoding problem in error-correcting codes. Hence, a conventional message modification and a proposed improved message modification including changing semi-neutral bits can be viewed as an error-correcting process for a non-linear code $S$ in $F$. More precisely, for a non-linear code $S$ in $F$, an error-correction can be achieved by manipulating control bits and semi-neutral bits.

\section{Analysis of the 58-Round SHA-1 Using the Improved Message Modification}

In this part, we detail the different steps of our technique for finding a collision on 58-round SHA-1. In Table 2, we give the sufficient conditions. Note that we have only quoted the sufficient conditions for the first 20 rounds due to space limitation. For the complete list of the conditions, see 910. The control bits and controlled relations are given in Table 3 . Semi-neutral bits and adjusters are given in Table 4.

- 'a' means $a_{i, j}=a_{i-1, j}$

- 'A' means $a_{i, j}=a_{i-1, j}+1$

- 'b' means $\left.a_{i, j}=a_{i-1,(j+2} \bmod 32\right)$

- 'B' means $\left.a_{i, j}=a_{i-1,(j+2} \bmod 32\right)+1$

- 'c' means $\left.a_{i, j}=a_{i-2,(j+2} \bmod 32\right)$

- 'C' means $a_{i, j}=a_{i-2,(j+2 \bmod 32)}+1$

- 'L' means the leading term of controlled relation of Table 3

- 'w', 'W': adjust $a_{i, j}$ so that $m_{i+1, j}=0,1$, respectively

- 'v', 'V': adjust $a_{i, j}$ so that $m_{i,(j+27 \bmod 32)}=0,1$, respectively

- 'h': adjust $a_{i, j}$ so that corresponding controlled relation including $m_{i+1, j}$ as leading term holds

- ' $r$ ' means to adjust $a_{i, j}$ so that corresponding controlled relation including $m_{i,(j+27 \bmod 32)}$ as leading term holds 
Table 2. Sufficient condition on the $m_{i, j}$ (resp. $\left.a_{i, j}\right)$
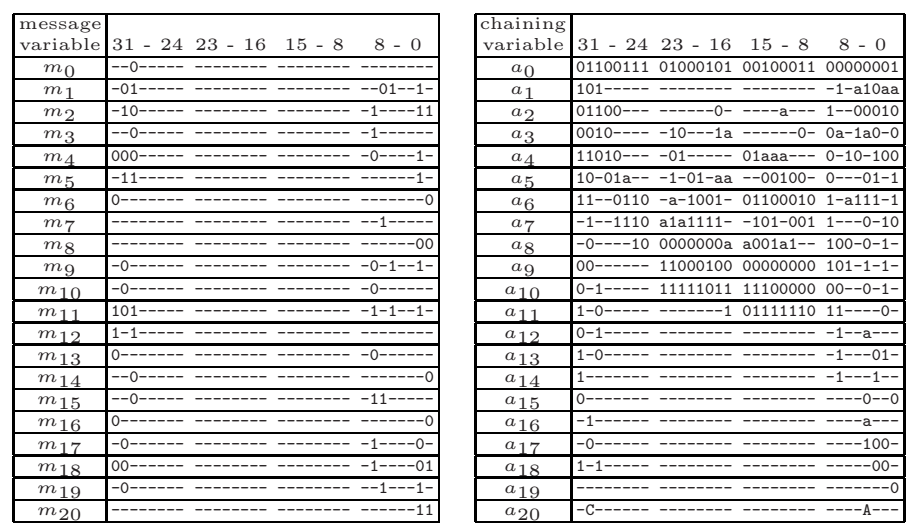

Table 3. Control bits and controlled relations

\begin{tabular}{|c|c|c|}
\hline \begin{tabular}{|c|} 
Control \\
sequence \\
$s_{i}$
\end{tabular} & $\begin{array}{c}\text { Control } \\
\text { bit } \\
b_{i} \\
\end{array}$ & Controlled relation $r_{i}$ \\
\hline$s_{124}$ & $a_{16,7}, a_{15,9}, a_{14,9}$ & $a_{23,0}=0$ \\
\hline$s_{123}$ & $a_{16,9}$ & $a_{22,2}+a_{21,2}=1$ \\
\hline$s_{122}$ & $a_{16,13}, a_{15,15}, a_{15,12}, a_{15,11}$ & $a_{22,1}=1$ \\
\hline$s_{121}$ & $a_{16,10}$ & $a_{21,3}+m_{20,3}=0$ \\
\hline$s_{120}$ & $a_{16,8}$ & $a_{21,1}=1$ \\
\hline$s_{119}$ & $a_{16,15, a_{16,20}}$ & $a_{20,3}+m_{19,3}=1$ \\
\hline$s_{118}$ & $a_{16,17}$ & $a_{19,0}=0$ \\
\hline$s_{117}$ & $a_{16,21}$ & $a_{18,31}=1$ \\
\hline$s_{116}$ & $a_{16,19}$ & $a_{18,29}=1$ \\
\hline$s_{115}$ & $a_{13,4}$ & $a_{18,2}=0$ \\
\hline$s_{114}$ & $a_{13,3}$ & $a_{18,1}=0$ \\
\hline$s_{113}$ & $a_{14,15}$ & $a_{17,30}=0$ \\
\hline$s_{112}$ & $a_{16,31}$ & $m_{15,31}=1$ \\
\hline$s_{111}$ & $a_{16,29}$ & $m_{15,29}=0$ \\
\hline$s_{110}$ & $a_{16,28}$ & $m_{15,28}+m_{10,28}+m_{8,29}+m_{7,29}+m_{4,28}+m_{2,28}=1$ \\
\hline$s_{109}$ & $a_{16,27}, a_{13,28}$ & $\begin{array}{l}m_{15,27}+m_{14,25}+m_{12,28}+m_{12,26}+m_{10,28}+m_{9,27}+m_{9,25}+m_{8,29}+ \\
m_{8,28}+m_{7,28}+m_{7,27}+m_{6,26}+m_{5,28}+m_{4,26}+m_{3,25}+m_{2,28}+m_{1,25}+ \\
m_{0,28}=1\end{array}$ \\
\hline$s_{108}$ & $a_{16,26}$ & $\begin{array}{l}m_{15,26}+m_{10,28}+m_{10,26}+m_{8,28}+m_{8,27}+m_{7,27}+m_{6,29}+m_{5,27}+ \\
m_{4,26}+m_{2,27}+m_{2,26}+m_{0,27}=1\end{array}$ \\
\hline${ }^{s_{107}}$ & $a_{16,25}$ & 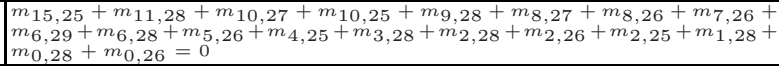 \\
\hline$s_{106}$ & $\overline{a_{16,24}}$ & $\begin{array}{l}m_{15,24+m_{12,28}+m_{11,27}+m_{10,26}+m_{10,24}+m_{9,28}+m_{9,27}+m_{8,29}+} m_{8,26}+m_{8,25}+m_{7,25}+m_{6,29}+m_{6,28}+m_{6,27}+m_{5,25}+m_{4,28}+m_{4,24}+ \\
m_{3,28}+m_{3,27}+m_{2,27}+m_{2,25}+m_{2,24}+m_{1,28}+m_{1,27}+m_{0,27}+m_{0,25}=1\end{array}$ \\
\hline$s_{105}$ & $a_{16,23}$ & 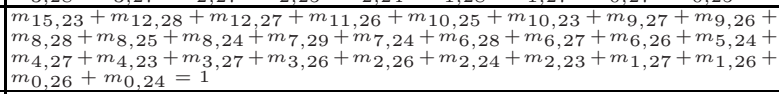 \\
\hline$s_{104}$ & $a_{16,22}$ & $\begin{array}{l}m_{15,22}+m_{14,25}+m_{12,28}+m_{12,27}+m_{11,25}+m_{10,27}+m_{10,24}+m_{10,22}+ \\
m_{9,28}+m_{9,27}+m_{9,26}+m_{8,27}+m_{8,24}+m_{8,23}+m_{7,28}+m_{7,27}+m_{7,23} \\
m_{6,27}+m_{6,25}+m_{5,23}+m_{4,28}+m_{4,27}+m_{4,22}+m_{3,26}+m_{2,28}+m_{2,27} \\
m_{2,25}+m_{2,23}+m_{2,22}+m_{1,26}+m_{0,25}+m_{0,23}=0\end{array}$ \\
\hline$s_{103}$ & $a_{16,6}$ & $m_{15,6}=1$ \\
\hline$s_{102}$ & $a_{16,5}$ & $m_{15,5}=1$ \\
\hline$s_{101}$ & $a_{16,4}$ & $0,4+m_{4,5}+m_{4,4}+m_{2,5}+m_{2,4}=1$ \\
\hline
\end{tabular}

\begin{tabular}{|l|l|l|}
\hline$s_{3}$ & $a_{1,28}$ & $m_{1,1}=1$ \\
\hline$s_{2}$ & $a_{1,25}$ & $m_{1,30}=0$ \\
\hline$s_{1}$ & $a_{1,24}$ & $m_{1,29}=1$ \\
\hline$s_{0}$ & $a_{1,23}$ & $m_{1,29}=1$ \\
\hline
\end{tabular}


Table 4. Semi-neutral bits and adjusters

\begin{tabular}{|c|c|c|c|}
\hline $\begin{array}{l}\text { message } \\
\text { variable }\end{array}$ & $31-2423-16$ & $15-8$ & $8-0$ \\
\hline$m_{0}$ & ---0----- -------- & -------- & $=--$ \\
\hline$m_{1}$ & $-01-----\quad--------$ & -------- & $--01--1-$ \\
\hline$m_{2}$ & L10----- -------- & -------- & $-1----11$ \\
\hline$m_{3}$ & -LO----- -------- & -------- & $-1------$ \\
\hline$m_{4}$ & $000-----\quad--------$ & -------- & $-0----1-$ \\
\hline$m_{5}$ & L11----- -------- & -------- & $------1 \mathrm{~L}$ \\
\hline$m_{6}$ & OL------ -------- & -------- & ---- \\
\hline$m_{7}$ & LL------ -------- & -------- & $--1----\mathrm{L}$ \\
\hline$m_{8}$ & LL------- --------- & -------- & ------00 \\
\hline$m_{9}$ & LOL----- -------- & -------- & $-0 \mathrm{~L} 1--1 \mathrm{~L}$ \\
\hline$m_{10}$ & LOL----- -------- & -------- & $-0 \mathrm{~L}----\mathrm{L}$ \\
\hline$m_{11}$ & 101----- --------- & -------- & $-1-1--1 \mathrm{~L}$ \\
\hline$m_{12}$ & 1L1----- --------- & -------- & $------\mathrm{L}$ \\
\hline$m_{13}$ & OLLLLL-L LL------ & -------- & -OLLLLLL \\
\hline$m_{14}$ & LLOLLL-L LLLL---- & -------- & --LLLLLO \\
\hline$m_{15}$ & LLOLLLLL LL------- & -------- & -11 LLLLL \\
\hline$m_{16}$ & 0 & -------- & -------0 \\
\hline$m_{17}$ & $-0------\quad-------$ & -------- & $-1----0-$ \\
\hline$m_{18}$ & $00--------------$ & -------- & $-1----01$ \\
\hline$m_{19}$ & $-0------\quad--------$ & -------- & $--1---1-$ \\
\hline$m_{20}$ & 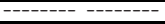 & -------- & ------11 \\
\hline$m_{21}$ & $-0------\quad--------$ & ---- & $-0----1-$ \\
\hline$m_{22}$ & 01------ -------- & -------- & $-0----10$ \\
\hline$m_{23}$ & 11------- --------- & -------- & $--1---0-$ \\
\hline$m_{24}$ & --------- -------- & --------- & -- \\
\hline$m_{25}$ & -1------ -------- & -------- & $-1-$ \\
\hline$m_{26}$ & $10------\quad---\cdots---$ & -------- & $-0----10$ \\
\hline$m_{27}$ & $\mid-1------\quad---------$ & -------- & $-01---0-$ \\
\hline$m_{28}$ & 1-------- --------- & -------- & -------0 \\
\hline$m_{29}$ & $-1------\quad--------$ & -------- & $-1----0-$ \\
\hline$m_{30}$ & 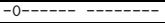 & -------- & $-1----0-$ \\
\hline$m_{31}$ & \begin{tabular}{|l|l|}
$-1------$ & -------- \\
\end{tabular} & -------- & $------0-$ \\
\hline$m_{32}$ & --------- --------- & ------- & $-----1-$ \\
\hline$m_{33}$ & 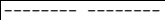 & ------- & $-0------$ \\
\hline$m_{34}$ & 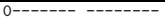 & ------- & $-----1-$ \\
\hline$m_{35}$ & 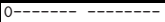 & ------- & -- \\
\hline$m_{36}$ & 1------- --------- & -------- & $------1-$ \\
\hline$m_{37}$ & $1--------\quad--------$ & -------- & $-0----$ \\
\hline$m_{38}$ & 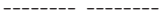 & -------- & --- \\
\hline$m_{39}$ & 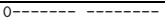 & ------- & $-1-----$ \\
\hline$m_{40}$ & 1------- -------- & -------- & -- \\
\hline$m_{41}$ & \begin{tabular}{|l|l}
-------- & -------- \\
\end{tabular} & -------- & $-1------$ \\
\hline$m_{42}$ & 1-------- --------- & --------- & --------- \\
\hline$m_{43}$ & |-------- --------- & --------- & $-1------$ \\
\hline$m_{44}$ & $1--------\quad--------$ & --------- & $------1-$ \\
\hline$m_{45}$ & -------- -------- & -------- & -- \\
\hline$m_{46}$ & 1------- -------- & -------- & -------- \\
\hline$m_{47}$ & 0------- - - - & -------- & ---- \\
\hline$n_{i}(i \geq 48)$ & ------ & -- & \\
\hline
\end{tabular}

\begin{tabular}{|c|c|c|c|}
\hline $\begin{array}{l}\text { chaining } \\
\text { variable }\end{array}$ & $31-2423-16$ & $15-8$ & $8-0$ \\
\hline$a_{0}$ & 0110011101000101 & 00100011 & 00000001 \\
\hline$a_{1}$ & $101 \mathrm{~V}--\mathrm{vV}$ Y-------- & -------- & $-1-a 10 a a$ \\
\hline$\overline{a_{2}}$ & $01100 \mathrm{vVv}$-------0- & $----a---$ & 1 -พ00010 \\
\hline$a_{3}$ & $0010--V v-10---1 a$ & $------0-$ & OaX1a0w0 \\
\hline$a_{4}$ & $11010 \mathrm{vv}--01-----$ & 01aaa--- & 0W10-100 \\
\hline$a_{5}$ & 10w01aV- -1-01-aa & $--00100-$ & 0 w--01W1 \\
\hline$a_{6}$ & $11 \mathrm{~W}-0110-a-1001-$ & 01100010 & 1-a111W1 \\
\hline$a_{7}$ & w1x-1110 a1a1111- & $-101-001$ & $1---0-10$ \\
\hline$a_{8}$ & h0Xvvv10 $0000000 \mathrm{a}$ & a001a1-- & $100 \times 0-1 \mathrm{~h}$ \\
\hline$a_{9}$ & $00 X V r r-V \quad 11000100$ & 00000000 & $101-1-1 \mathrm{y}$ \\
\hline$a_{10}$ & 0w1-rv-v 11111011 & 11100000 & $00 \mathrm{hWO}-1 \mathrm{~h}$ \\
\hline$a_{11}$ & $1 \mathrm{w} 0--\mathrm{V}-\mathrm{V} \quad-------1$ & 01111110 & $11 \mathrm{x}^{---}-0 \mathrm{Y}$ \\
\hline$a_{12}$ & 0w1-rV-V -------- & -------- & $-1 \mathrm{XWa}-\mathrm{Wh}$ \\
\hline$a_{13}$ & 1w0--vv- -rr------ & -------- & $-1-\mathrm{qq} 01 \mathrm{y}$ \\
\hline$a_{14}$ & 1rhhvvVh hh------- & qNNNNNqN & N1hhh1hh \\
\hline$a_{15}$ & OrwhhhVh hhhh---N & qNNqqNqN & NNhhOhh0 \\
\hline$a_{16}$ & W1whhhhh hhqNqNqN & NNqNNqqq & qWWhahhh \\
\hline$a_{17}$ & $-0--------------$ & -------- & $----100-$ \\
\hline$a_{18}$ & $1-1-------------$ & -------- & $-----00-$ \\
\hline$a_{19}$ & -------- -------- & ------- & -------0 \\
\hline$a_{20}$ & -C------- -------- & -------- & $----A---$ \\
\hline$a_{21}$ & -b------- --------- & -------- & $----a-1-$ \\
\hline$a_{22}$ & 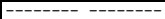 & ------- & $-----\mathrm{A} 1-$ \\
\hline$a_{23}$ & |-------- -------- & -------- & -------0 \\
\hline$a_{24}$ & |-c------ -------- & -------- & ----1 \\
\hline$a_{25}$ & 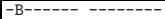 & ------- & $----a-$ \\
\hline$a_{26}$ & 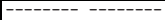 & -------- & $----\mathrm{A} 1-$ \\
\hline$a_{27}$ & --------- -------- & --------- & -------1 \\
\hline$a_{28}$ & -c------- --------- & -------- & $----A---$ \\
\hline$a_{29}$ & -B------- --------- & -------- & $----A-0-$ \\
\hline$a_{30}$ & --------- --------- & ------- & $-----0-$ \\
\hline$a_{31}$ & |-------- -------- & --------- & -------- \\
\hline$a_{32}$ & |-------- --------- & --------- & $----A---$ \\
\hline$a_{33}$ & -------- ------- & -------- & $------1-$ \\
\hline$a_{34}$ & - - - - - - - - - - & -------- & -------- \\
\hline$a_{35}$ & - - - - - - - - - - & -------- & -- \\
\hline$a_{36}$ & |--------- --------- & -------- & $----A---$ \\
\hline$a_{37}$ & |-------- -------- & -------- & $------1-$ \\
\hline$a_{38}$ & |-----o--- --------- & -------- & $----A---$ \\
\hline$a_{39}$ & B-------- --------- & --------- & $-------0-$ \\
\hline$a_{40}$ & C-------- --------- & -------- & $----A---$ \\
\hline$a_{41}$ & B-------- -------- & -------- & $------0-$ \\
\hline$a_{42}$ & C-------- --------- & --------- & $----A--$ \\
\hline$a_{43}$ & B-------- --------- & --------- & $--0-$ \\
\hline$a_{44}$ & C-------- --- & --- & \\
\hline$a_{45}$ & B-------- -------- & -------- & --- \\
\hline$(i \geq 46$ & ---------- --------- & --------- & ------- \\
\hline
\end{tabular}

- 'x', 'y': adjust $a_{i+1, j-1}, a_{i, j-1}$ so that $m_{i, j}=0$, respectively

- 'X', 'Y': adjust $a_{i+1, j-1}, a_{i, j-1}$ so that $m_{i, j}=1$, respectively

- 'N': semi-neutral bit

- 'q' : adjust $a_{i, j}$ so that relations after 17 -round hold

In this case, the set of bits corresponding to ' $q$ ' is exactly same to the set of adjusters.

The conditions remaining after the conventional message modification are listed below: $a_{17,3}=1, a_{17,2}=0, a_{17,1}=0, a_{26,1}=1, a_{27,0}=1, a_{29,1}=0, a_{30,1}=$ $0, a_{33,1}=1, a_{37,1}=1, a_{39,1}=0, a_{41,1}=0, a_{43,1}=0, a_{20,30}+a_{18,0}=1, a_{21,30}+$ $a_{20,0}=0, a_{24,30}+a_{22,0}=0, a_{25,30}+a_{24,0}=1, a_{25,3}+a_{24,3}=0, a_{26,2}+a_{25,2}=$ $1, a_{28,30}+a_{26,0}=0, a_{28,3}+a_{27,3}=1, a_{29,30}+a_{28,0}=1, a_{29,3}+a_{28,3}=1, a_{32,3}+$ $a_{31,3}=1, a_{36,3}+a_{35,3}=1, a_{38,3}+a_{37,3}=1, a_{39,31}+a_{38,1}=1, a_{40,3}+a_{39,3}=$ $1, a_{40,31}+a_{38,1}=1, a_{41,31}+a_{40,1}=1, a_{42,31}+a_{40,1}=1, a_{43,31}+a_{42,1}=1, a_{42,3}+$ $a_{41,3}=1, a_{44,31}+a_{42,1}=1, a_{45,31}+a_{44,1}=1$. 
There are five conditions $a_{17,3}=1, a_{17,2}=0, a_{17,1=0}, a_{20,30}+a_{18,0}=1, a_{21,30}+$ $a_{20,0}=0$ which are only related to first 23 rounds. For other 29 conditions, we adjust by using 21 semi-neutral bits and 11 adjusters as explained in the improved message modification algorithm (see Algorithm 21).

\section{A Concluding Note}

This paper present an improved method for finding collision on SHA-1. To do so, we use algebraic techniques for describing the message modification technique and propose an improvement. The details of our attack can be found in the appendices. The proposed method improves the complexity of an attack against 58-round SHA-1 and we found many new collisions.

\section{References}

1. Biham, E., Chen, R., Joux, A., Carribault, P., Lemuet, C., Jalby, W.: Collisions of SHA-0 and Reduced SHA-1. In: Cramer, R.J.F. (ed.) EUROCRYPT 2005. LNCS, vol. 3494, pp. 36-57. Springer, Heidelberg (2005)

2. Biham, E., Chen, R.: Near-Collisions of SHA-0. In: Franklin, M. (ed.) CRYPTO 2004. LNCS, vol. 3152, pp. 290-305. Springer, Heidelberg (2004)

3. De Cannière, C., Rechberger, C.: Finding SHA-1 Characteristics. In: Lai, X., Chen, K. (eds.) ASIACRYPT 2006. LNCS, vol. 4284, pp. 1-20. Springer, Heidelberg (2006)

4. Chabaud, F., Joux, A.: Differential Collisions in SHA-0. In: Krawczyk, H. (ed.) CRYPTO 1998. LNCS, vol. 1462, pp. 56-71. Springer, Heidelberg (1998)

5. Dobbertin, H.: Cryptanalysis of MD4. In: Gollmann, D. (ed.) Fast Software Encryption. LNCS, vol. 1039, pp. 53-69. Springer, Heidelberg (1996)

6. Hui, L.C.K., Wang, X., Chow, K.P., Tsang, W.W., Chong, C.F., Chan, H.W.: The Differential Analysis of Skipjack Variants from the first Round. In: Advance in Cryptography - CHINACRYPT 2002 Science Publishing House (2002)

7. Joux, A.: Multicollisions in Iterated Hash Functions. Application to Cascaded Constructions. In: Franklin, M. (ed.) CRYPTO 2004. LNCS, vol. 3152, pp. 306-316. Springer, Heidelberg (2004)

8. Pramstaller, N., Rechberger, C., Rijmen, V.: Exploiting Coding Theory for Collision Attacks on SHA-1. In: Smart, N.P. (ed.) Cryptography and Coding. LNCS, vol. 3796, pp. 78-95. Springer, Heidelberg (2005)

9. Sugita, M., Kawazoe, M., Imai, H.: Gröbner Basis Based Cryptanalysis of SHA-1. IACR Cryptology ePrint Archive 2006/098 (2006), http://eprint.iacr.org/2006/098.pdf

10. Sugita, M., Kawazoe, M., Imai, H.: Gröbner Basis Based Cryptanalysis of SHA-1. In: Proc. of SECOND NIST CRYPTOGRAPHIC HASH WORKSHOP (2006)

11. Wang, X., Lai, X., Feng, D., Chen, H., Yu, X.: Cryptanalysis of the Hash Functions MD4 and RIPEMD. In: Cramer, R.J.F. (ed.) EUROCRYPT 2005. LNCS, vol. 3494, pp. 1-18. Springer, Heidelberg (2005)

12. Wang, X., Feng, D., Yu, X.: An Attack on Hash Function HAVAL-128. Science in China Series 48, 545-556 (2005)

13. Wang, X., Yao, A.C., Yao, F.: Cryptanalysis on SHA-1. In: Proc. of NIST Cryptographic Hash Workshop (2005) 
14. Wang, X., Yin, Y.L., Yu, H.: New Collision Search for SHA-1. In: Rump Session of CRYPTO (2005)

15. Wang, X., Yin, Y.L., Yu, H.: Finding Collisions in the Full SHA-1. In: Shoup, V. (ed.) CRYPTO 2005. LNCS, vol. 3621, pp. 17-36. Springer, Heidelberg (2005)

16. Wang, X., Yu, H.: How to Break MD5 and Other Hash Functions. In: Cramer, R.J.F. (ed.) EUROCRYPT 2005. LNCS, vol. 3494, pp. 19-35. Springer, Heidelberg (2005)

17. Wang, X., Yu, H., Yin, Y.L.: Efficient Collision Search Attacks on SHA-0. In: Shoup, V. (ed.) CRYPTO 2005. LNCS, vol. 3621, pp. 1-16. Springer, Heidelberg (2005)

18. Wang, X.: The Collision attack on SHA-0 (1997)

19. Wang, X.: The Improved Collision attack on SHA-0 (1998)

20. Wang, X.: Collisions for Some Hash Functions MD4, MD5, HAVAL-128, RIPEMD. In: Rump Session of Crypto'04

21. Wang, X.: Cryptanalysis of Hash Functions and Potential Dangers. In: RSA Conference 2006, San Jose, USA (2006)

22. Klima, V.: Tunnels in Hash Functions: MD5 Collisions Within a Minute. IACR Cryptology ePrint Archive 2006/105 (2006), http://eprint.iacr.org/ 2006/098.pdf

\section{Appendix}

\section{A Analysis of the Full SHA-1}

We present here the details of our method on the full SHA-1.

\section{A.1 Disturbance Vector and Differential}

We start from the disturbance vector and differential given by Wang et al [13. From these, we construct a new differential. We modify Wang's differential by

Table 5. A differential for the full SHA-1

\begin{tabular}{|c|ccc|}
\hline & $\Delta m$ & $\Delta^{+} m$ & $\Delta^{-} m$ \\
\hline$i=0$ & $a 0000003$ & 00000001 & $a 0000002$ \\
\hline$i=1$ & 20000030 & 20000020 & 00000010 \\
\hline$i=2$ & 60000000 & 60000000 & 00000000 \\
\hline$i=3$ & $e 000002 a$ & 40000000 & $a 000002 a$ \\
\hline$i=4$ & 20000043 & 20000042 & 00000001 \\
\hline$i=5$ & 60000040 & $a 0000000$ & 10000040 \\
\hline$i=6$ & $d 0000053$ & $d 0000042$ & 00000011 \\
\hline$i=7$ & $d 0000022$ & $d 0000000$ & 00000022 \\
\hline$i=8$ & 20000000 & 00000000 & 20000000 \\
\hline$i=9$ & 60000032 & 20000030 & 40000002 \\
\hline$i=10$ & 60000043 & 60000041 & 00000002 \\
\hline$i=11$ & 20000040 & 00000000 & 20000040 \\
\hline$i=12$ & $e 0000042$ & $c 0000000$ & 20000042 \\
\hline$i=13$ & 60000002 & 00000002 & 60000000 \\
\hline$i=14$ & 80000001 & 00000001 & 80000000 \\
\hline$i=15$ & 00000020 & 00000020 & 00000000 \\
\hline$i=16$ & 00000003 & 00000002 & 00000001 \\
\hline$i=17$ & 40000052 & 00000002 & 40000050 \\
\hline$i=18$ & 40000040 & 00000000 & 40000040 \\
\hline$i=19$ & $e 0000052$ & 00000002 & $e 0000050$ \\
\hline$i=20$ & $a 0000000$ & 00000000 & $a 0000000$ \\
\hline
\end{tabular}

\begin{tabular}{|c|ccc|}
\hline & $\Delta a$ & $\Delta^{+} a$ & $\Delta^{-} a$ \\
\hline$i=0$ & 00000000 & 00000000 & 00000000 \\
\hline$i=1$ & $e 0000001$ & $a 0000000$ & 40000001 \\
\hline$i=2$ & 20000004 & 20000000 & 00000004 \\
\hline$i=3$ & $c 07 f f f 84$ & $803 f f f 84$ & 40400000 \\
\hline$i=4$ & $800030 e 2$ & $800010 a 0$ & 00002042 \\
\hline$i=5$ & $084080 b 0$ & 08008020 & 00400090 \\
\hline$i=6$ & $80003 a 00$ & $00001 a 00$ & 80002000 \\
\hline$i=7$ & $0 f f f 8001$ & 08000001 & $07 f f 8000$ \\
\hline$i=8$ & 00000008 & 00000008 & 00000000 \\
\hline$i=9$ & 80000101 & 80000100 & 00000001 \\
\hline$i=10$ & 00000002 & 00000002 & 00000000 \\
\hline$i=11$ & 00000100 & 00000000 & 00000100 \\
\hline$i=12$ & 00000002 & 00000002 & 00000000 \\
\hline$i=13$ & 00000000 & 00000000 & 00000000 \\
\hline$i=14$ & 00000000 & 00000000 & 00000000 \\
\hline$i=15$ & 00000001 & 00000001 & 00000000 \\
\hline$i=16$ & 00000000 & 00000000 & 00000000 \\
\hline$i=17$ & 80000002 & 80000002 & 00000000 \\
\hline$i=18$ & 00000002 & 00000002 & 00000000 \\
\hline$i=19$ & 80000002 & 80000002 & 00000000 \\
\hline$i=20$ & 00000000 & 00000000 & 00000000 \\
\hline
\end{tabular}


changing $\Delta a_{14,31}^{+}$and $\Delta a_{14,31}^{-}$. For our method, it is important to choose $\Delta^{+} m$, $\Delta^{-} m, \Delta^{+} a$ and $\Delta^{-} a$ carefully. A bad choice of plus/minus could lead to an underdefined system of equations (as result of the Gaussian elimination). For this reason, we have to correct Wang's differential. We present in Table [5 the new differential constructed.

\section{A.2 Sufficient Conditions}

For the disturbance vector, and the differential given in the previous step, we give in Table 6 the sufficient conditions for the full SHA-1. In Table 6. 'a' means $a_{i, j}=a_{i-1, j}$, 'A' means $a_{i, j}=a_{i-1, j}+1$, 'b' means $\left.a_{i, j}=a_{i-1,(j+2} \bmod 32\right)$, 'B' means $a_{i, j}=a_{i-1,(j+2 \bmod 32)}+1$, 'c' means $a_{i, j}=a_{i-2,(j+2 \bmod 32)}$ and 'C' means $a_{i, j}=a_{i-2,(j+2 \bmod 32)}+1$.

Table 6. Sufficient conditions for the full SHA-1

\begin{tabular}{|c|c|c|c|}
\hline $\begin{array}{l}\text { message } \\
\text { variable }\end{array}$ & $31-2423-16$ & $15-8$ & $8-0$ \\
\hline$m_{0}$ & $11-1-------$ & --- & $\frac{-1}{-1}$ \\
\hline$m_{1}$ & $-0------1$ & & --01 \\
\hline$m_{2}$ & $00------$ & & \\
\hline$m_{3}$ & 101----- -------- & $\overline{---}$ & $--1-1-1-$ \\
\hline$m_{4}$ & $--0-------$ & --- & $-0----01$ \\
\hline$m_{5}$ & 0-01----- -------- & -------- & $-1--$ \\
\hline 6 & $00-0----\quad---\cdot---$ & -------- & $-0-1--01$ \\
\hline 7 & $00-0------\cdot----$ & -------- & $--1---1-$ \\
\hline$m_{8}$ & $\mid--1---------$ & -------- & --- \\
\hline$m_{9}$ & $-10--------$ & -------- & $--00--1-$ \\
\hline$m_{10}$ & $\mid-00-------\cdot-----$ & -------- & $-0----10$ \\
\hline$m_{11}$ & |--1------ --------- & --------- & $-1--$ \\
\hline$m_{12}$ & 001----- -------- & --------- & $-1----1-$ \\
\hline$m_{13}$ & -11----- -------- & -------- & $------0-$ \\
\hline 4 & 1-------- -------- & -------- & -------0 \\
\hline$m_{15}$ & 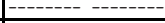 & -------- & $--0---$ \\
\hline$m_{16}$ & -------- -------- & --- & ------01 \\
\hline$m_{17}$ & $\mid-1-------\quad--------$ & $=--$ & $-1-1--0-$ \\
\hline$m_{18}$ & $\mid-1-------\quad--------1$ & --- & $-1----$ \\
\hline$m_{19}$ & $111-------$ & $\cdots$ & $-1-1--0-$ \\
\hline$m_{20}$ & 1 & & \\
\hline
\end{tabular}

\begin{tabular}{|c|c|c|c|}
\hline $\begin{array}{l}\text { haining } \\
\text { ariable }\end{array}$ & $31-2423-16$ & $15-8$ & $8-0$ \\
\hline$\overline{a_{0}}$ & $\begin{array}{|ll|}01100111 & 01000101\end{array}$ & 00100011 & 00000001 \\
\hline 1 & $010----0 \quad-0-01-0-$ & $10-0-10-$ & $-\mathrm{a} 0101$ \\
\hline$\overline{a_{2}}$ & $-100---1$ 0aa10a1a & $01 \mathrm{a} 1 \mathrm{a} 011$ & 1--a11a1 \\
\hline$\overline{a_{3}}$ & $01011----1000000$ & 00000000 & $01--\mathrm{a} 0 \mathrm{a} 1$ \\
\hline$a_{4}$ & $0-101--a---10000$ & 00101000 & $010---10$ \\
\hline$a_{5}$ & $0-0101-1-1-11110$ & $00111-00$ & 10010100 \\
\hline$a_{6}$ & 1-0a1a0a a0a1aaa- & $--10010-$ & $--01-0--$ \\
\hline$a_{7}$ & $--0-011111111111$ & 111-010- & $0-0-0110$ \\
\hline$\overline{a_{8}}$ & $-10---01 \quad 11110000$ & $010-111-$ & $1---000-$ \\
\hline$a_{9}$ & $00----11 \quad 11111111$ & $111----0$ & $---1-01$ \\
\hline$a_{10}$ & -11----- --------- & $-----a--$ & $-1--1-0-$ \\
\hline$a_{11}$ & $100-----\quad--------$ & -------1 & $-1--0---$ \\
\hline$a_{12}$ & $---------\quad---$ & $=-$ & $-1----0-$ \\
\hline$a_{13}$ & $0---------$ & --------- & $-1---0--$ \\
\hline$a_{14}$ & 1------- --------- & --------- & $-----1--$ \\
\hline$a_{15}$ & ----------- & -- & $--0--0$ \\
\hline$a_{16}$ & $-1------\quad----$ & -- & $--1-A-$ \\
\hline$a_{17}$ & $00-------\quad----$ & -- & $----0-0-$ \\
\hline$a_{18}$ & 11-1----- --------- & -------- & $----a-0-$ \\
\hline$a_{19}$ & 0-b----- -------- & ------- & $-----0-$ \\
\hline$a_{20}$ & --- & & \\
\hline
\end{tabular}

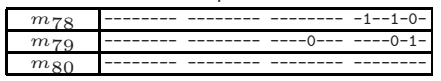

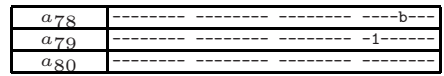

By a Gaussian elimination, we reduce all conditions on the $m_{i}$. The result of Gaussian elimination is listed below. There are 167 conditions on $m_{i, j}$ :

\footnotetext{
$m_{15,31}=0, m_{15,30}=1, m_{15,29}=1, m_{15,28}+m_{10,28}+m_{4,28}+m_{2,28}=0, m_{15,27}+m_{10,27}+m_{8,28}+$ $m_{4,27}+m_{2,28}+m_{2,27}+m_{0,28}=1, m_{15,26}+m_{10,28}+m_{10,26}+m_{8,28}+m_{8,27}+m_{7,27}+m_{5,27}+m_{4,26}+$ $m_{2,27}+m_{2,26}+m_{0,27}=0, m_{15,25}+m_{11,28}+m_{10,27}+m_{10,25}+m_{9,28}+m_{8,27}+m_{8,26}+m_{7,26}+m_{5,26}+$ $m_{4,25}+m_{3,28}+m_{2,28}+m_{2,26}+m_{2,25}+m_{1,28}+m_{0,28}+m_{0,26}=0, m_{15,24}+m_{12,28}+m_{11,27}+m_{10,26}+$ $m_{10,24}+m_{9,28}+m_{9,27}+m_{8,26}+m_{8,25}+m_{7,25}+m_{6,27}+m_{5,25}+m_{4,28}+m_{4,24}+m_{3,28}+m_{3,27}+m_{2,27}+$ $m_{2,25}+m_{2,24}+m_{1,28}+m_{1,27}+m_{0,27}+m_{0,25}=1, m_{15,23}+m_{12,28}+m_{12,27}+m_{11,26}+m_{10,25}+m_{10,23}+$ $m_{9,27}+m_{9,26}+m_{8,28}+m_{8,25}+m_{8,24}+m_{7,24}+m_{7,0}+m_{6,27}+m_{6,26}+m_{5,24}+m_{4,27}+m_{4,23}+m_{3,27}+$ $m_{3,26}+m_{2,26}+m_{2,24}+m_{2,23}+m_{1,30}+m_{1,27}+m_{1,26}+m_{1,0}+m_{0,26}+m_{0,24}=0$,
}

$m_{5,0}+m_{1,30}+m_{1,0}=0, m_{4,31}=0, m_{4,30}=0, m_{4,29}=0, m_{4,6}=0, m_{4,1}=0, m_{4,0}=1, m_{3,31}=1$, $m_{3,30}=0, m_{3,29}=1, m_{3,5}=1, m_{3,3}=1, m_{3,1}=1, m_{3,0}+m_{1,0}=0, m_{2,31}=1, m_{2,30}=0, m_{2,29}=0$, $m_{2,0}+m_{1,30}=1, m_{1,31}+m_{1,30}=1, m_{1,29}=0, m_{1,5}=0, m_{1,4}=1, m_{0,31}=1, m_{0,30}=0, m_{0,29}=1$, $m_{0,1}=1, m_{0,0}=0$. 


\section{A.3 Control Bits and Controlled Relations}

The control bits and controlled relations are presented in Table 7 .

Now we give the semi-neutral bits and adjuster in Table 8 In this table :

- 'a', 'A', 'b', 'B', 'c', 'C': as in Section A.2.

- 'L' means the leading term of controlled relation of Table 7.

- 'w', 'W': adjust $a_{i, j}$ so that $m_{i+1, j}=0,1$, respectively.

- 'v', 'V': adjust $a_{i, j}$ so that $\left.m_{i,(j+27} \bmod 32\right)=0,1$, respectively.

- ' $\mathrm{h}$ ': adjust $a_{i, j}$ so that corresponding controlled relation including $m_{i+1, j}$ as leading term holds.

- ' $r$ ' means to adjust $a_{i, j}$ so that corresponding controlled relation including $\left.m_{i,(j+27} \bmod 32\right)$ as leading term holds.

- 'x', 'y': adjust $a_{i+1, j-1}, a_{i, j-1}$ so that $m_{i, j}=0$, respectively.

- 'X', 'Y': adjust $a_{i+1, j-1}, a_{i, j-1}$ so that $m_{i, j}=1$, respectively.

- 'N': semi-neutral bit.

- 'q' : adjust $a_{i, j}$ so that relations after 17 -round hold.

- 'F' : etc.

Table 7. Control bits and controlled relations for the full SHA-1

\begin{tabular}{|c|c|c|}
\hline ctrl. seq. & \begin{tabular}{|l|} 
control bits \\
\end{tabular} & \begin{tabular}{|c|} 
controlled relation \\
\end{tabular} \\
\hline$s_{168}$ & $a_{15,8}$ & $a_{30,2}+a_{29,2}=1$ \\
\hline$s_{167}$ & $a_{16,6}$ & $a_{26,2}+a_{25,2}=1$ \\
\hline$s_{166}$ & $a_{15,7}$ & $a_{25,3}+a_{24,3}=0$ \\
\hline$s_{165}$ & $a_{13,7}$ & $a_{24,3}+a_{23,3}=0$ \\
\hline$s_{164}$ & $a_{13,9}$ & $a_{23,0}=0$ \\
\hline$s_{163}$ & $a_{16,10}$ & $a_{22,3}+a_{21,3}=0$ \\
\hline$s_{162}$ & $a_{16,11}$ & $a_{21,29}+a_{20,31}=0$ \\
\hline$s_{161}$ & $a_{16,8}$ & $a_{21,1}=0$ \\
\hline$s_{160}$ & $a_{16,9}$ & $a_{20,29}=0$ \\
\hline$s_{159}$ & $a_{15,10}$ & $a_{20,3}+a_{19,3}=0$ \\
\hline$s_{158}$ & $a_{15,11}$ & $a_{19,31}=0$ \\
\hline$s_{157}$ & $a_{15,9}$ & $a_{19,29}+a_{18,31}=0$ \\
\hline$s_{156}$ & $a_{14,8}$ & $a_{19,1}=0$ \\
\hline$s_{155}$ & $a_{14,11}$ & $a_{18,31}=1$ \\
\hline$s_{154}$ & $a_{15,14}$ & $a_{18,29}=1$ \\
\hline$s_{153}$ & $a_{13,8}$ & $a_{18,1}=0$ \\
\hline$s_{152}$ & $a_{13,11}$ & $a_{17,31}=0$ \\
\hline$s_{151}$ & $a_{13,10}$ & $a_{17,30}=0$ \\
\hline$s_{150}$ & $a_{13,13}$ & $a_{17,1}=0$ \\
\hline$s_{149}$ & $a_{16,31}$ & $m_{15,31}=0$ \\
\hline$s_{148}$ & $a_{16,29}$ & $m_{15,29}=1$ \\
\hline$s_{147}$ & $a_{16,28}$ & $m_{15,28}+m_{10,28}+m_{4,28}+m_{2,28}=0$ \\
\hline$s_{146}$ & $a_{16,27}$ & $m_{15,27}+m_{10,27}+m_{8,28}+m_{4,27}+m_{2,28}+m_{2,27}+m_{0,28}=1$ \\
\hline$s_{145}$ & $a_{16,26}$ & $\begin{array}{l}m_{15,26}+m_{10,28}+m_{10,26}+m_{8,28}+m_{8,27}+m_{7,27}+m_{5,27}+m_{4,26}+m_{2,27}+m_{2,26}+ \\
m_{0,27}=0\end{array}$ \\
\hline$s_{144}$ & $a_{16,25}$ & $\begin{array}{l}m_{15,25}+m_{11,28}+m_{10,27}+m_{10,25}+m_{9,28}+m_{8,27}+m_{8,26}+m_{7,26}+m_{5,26}+ \\
m_{4,25}+m_{3,28}+m_{2,28}+m_{2,26}+m_{2,25}+m_{1,28}+m_{0,28}+m_{0,26}=0\end{array}$ \\
\hline$s_{143}$ & $a_{16,24}$ & $\begin{array}{l}m_{15,24}+m_{12,28}+m_{11,27}+m_{10,26}+m_{10,24}+m_{9,28}+m_{9,27}+m_{8,26}+m_{8,25}+ \\
m_{7,25}+m_{6,27}+m_{5,25}+m_{4,28}+m_{4,24}+m_{3,28}+m_{3,27}+m_{2,27}+m_{2,25}+m_{2,24}+ \\
m_{1,28}+m_{1,27}+m_{0,27}+m_{0,25}=1\end{array}$ \\
\hline$s_{142}$ & $a_{16,23}$ & $\begin{array}{l}m_{15,23}+m_{12,28}+m_{12,27}+m_{11,26}+m_{10,25}+m_{10,23}+m_{9,27}+m_{9,26}+m_{8,28}+ \\
m_{8,25}+m_{8,24}+m_{7,24}+m_{7,0}+m_{6,27}+m_{6,26}+m_{5,24}+m_{4,27}+m_{4,23}+m_{3,27}+ \\
m_{3,26}+m_{2,26}+m_{2,24}+m_{2,23}+m_{1,30}+m_{1,27}+m_{1,26}+m_{1,0}+m_{0,26}+m_{0,24}=0\end{array}$ \\
\hline
\end{tabular}

\begin{tabular}{|l|c|l|}
\hline$s_{3}$ & $a_{2,5}$ & $m_{1,5}=0$ \\
\hline$s_{2}$ & $a_{1,26}$ & $m_{1,31}+m_{1,30}=1$ \\
\hline$s_{1}$ & $a_{1,23}$ & $m_{1,29}=0$ \\
\hline
\end{tabular}


Table 8. Semi-neutral bits and Adjusters

\begin{tabular}{|c|c|c|c|}
\hline $\begin{array}{l}\text { message } \\
\text { variable }\end{array}$ & $31-2423-16$ & $15-8$ & $8-0$ \\
\hline$m_{0}$ & $1-1--------$ & -------- & ------10 \\
\hline$m_{1}$ & L-0----- --------- & -------- & $--01--$ \\
\hline$m_{2}$ & LOO----- -------- & -------- & ------1 \\
\hline$m_{3}$ & $101--------------$ & -------- & $--1-1-1 \mathrm{~L}$ \\
\hline$m_{4}$ & LLO------ --------- & -------- & $-0----01$ \\
\hline$m_{5}$ & L01---- -------- & -------- & $-1-----\mathrm{L}$ \\
\hline$m_{6}$ & LLO---- -------- & -------- & $-0-1--01$ \\
\hline$m_{7}$ & $00-0----\quad-------$ & -------- & $--1 \mathrm{~L}^{--1-}$ \\
\hline$m_{8}$ & L-1----- --------- & --------- & $----\mathrm{L}--\mathrm{L}$ \\
\hline$m_{9}$ & L10------ -------- & -------- & $--00-\mathrm{L} 1 \mathrm{~L}$ \\
\hline$m_{10}$ & LO0----- --------- & --------- & -0LLLL10 \\
\hline$m_{1}$ & LL1----- --------- & -------- & -1LLLLLL \\
\hline$m_{12}$ & $001-----\quad--------$ & -------- & $-1 \mathrm{LLL}-1 \mathrm{~L}$ \\
\hline$m]$ & L11LLLLL LLLLLLLL & L-L----- & --LLLLOL \\
\hline$m_{14}$ & 1LLLLLLL LLLLLLLL & L-LL---- & --LLLLLO \\
\hline$m_{15}$ & LLLLLLLL LLLLLLLL & LL-L---- & L-OLLLI \\
\hline$m_{16}$ & ----------- & -------- & -----01 \\
\hline 217 & 1------ -------- & -------- & $-1-1--4$ \\
\hline$m_{18}$ & $1------------\cdot--$ & -------- & $-1--$ \\
\hline$m_{1}$ & 111----- --------- & --------- & $-1-1--$ \\
\hline 170 & |-1----- -------- & -------- & ----- \\
\hline$m_{21}$ & ------ -------- & -------- & $-1----$ \\
\hline$m_{22}$ & -1----- --------- & --------- & ------ \\
\hline$m_{23}$ & -1----- --------- & -------- & $-11---$ \\
\hline 224 & P----- -------- & -------- & ------ \\
\hline$u_{25}$ & [------ --------- & --- & $-1----$ \\
\hline$m_{26}$ & 10------- -------- & --- & $-0----1$ \\
\hline$m_{2}$ & $1-------\cdots+---$ & +- & $--1---$ \\
\hline$m_{2}$ & P----- -------- & -- & ------1 \\
\hline$m_{29}$ & -0------- -------- & --- & $-0----0$ \\
\hline$m_{30}$ & 11------ -------- & --- & $-1----01$ \\
\hline$m_{31}$ & 11------- --------- & --------- & $--0---$ \\
\hline$m_{32}$ & P----- -------- & --- & ---- \\
\hline 233 & 0------ -------- & -------- & $-\cdots---0$ \\
\hline$m_{34}$ & O0------ ------- & -------- & $-1----00$ \\
\hline$m_{35}$ & - $0------\quad-------$ & ------- & $-11---1-$ \\
\hline 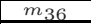 & 1------- -------- & -------- & -------0 \\
\hline 7 & |-1------ ---C---- & -------- & $-0----0-$ \\
\hline$m_{38}$ & - $0------\quad-\cdots-\cdots$ & -------- & $-1----1-$ \\
\hline 39 & $\mid-1--\cdot------$ & --- & $------0-$ \\
\hline 40 & - & -- & \\
\hline 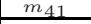 & $=$ & & -0 \\
\hline 42 & - & -- & \\
\hline$m$ & - n-- n & --- & \\
\hline 4 & - & $-\cdots$ & $-\cdots$ \\
\hline$n$ & - n-c- & - & $-1-$ \\
\hline 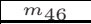 & -- & -- & \\
\hline 7 & -- & & $-1-$ \\
\hline$m_{48}$ & 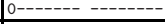 & $=--$ & - \\
\hline$m_{49}$ & ------ -------- & -------- & $-1----$ \\
\hline$m_{50}$ & ------ -------- & --------- & ------ \\
\hline$m_{51}$ & $-------\quad--------$ & -------- & $-1-----$ \\
\hline$m_{52}$ & ------- -------- & --------- & ------1 \\
\hline$m_{53}$ & ----- & -- & $-\cdots$ \\
\hline$m_{54}$ & ------ --- & $=--$ & - \\
\hline$m_{55}$ & ------ --- & - & \\
\hline$\underline{n_{5}}$ & ---- & -- & \\
\hline$m_{57}$ & - & --- & -- \\
\hline$m_{5}$ & ----- --- & --- & --- \\
\hline$m_{50}$ & --- & -- & \\
\hline$m_{60}$ & $=-$ & & \\
\hline$m_{61}$ & - & & \\
\hline 262 & ---- & $=-$ & \\
\hline 263 & ---- & -- & \\
\hline 6 & $=-$ & & \\
\hline$m_{65}$ & ------- ---- & --- & ---- \\
\hline 6 & ----- & -- & --0 \\
\hline 26 & ----- & -- & $1---$ \\
\hline 6 & - & $\cdots$ & - \\
\hline 69 & -- --------- & -------- & $----0--0$ \\
\hline$m_{70}$ & ------ -------- & ------1 & -------0 \\
\hline$m_{71}$ & ------- -------- & --------- & $----0--1$ \\
\hline$m_{72}$ & 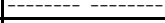 & $-\cdots-\cdots$ & $---0--0-$ \\
\hline$n_{73}$ & 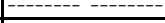 & $-\cdots---1-$ & $--\cdot---0-$ \\
\hline$n_{74}$ & $=$ & --- & $---00-1-$ \\
\hline 275 & -- & $-\cdots--1$ & $--1--0-$ \\
\hline 276 & - & $0--$ & ----00 \\
\hline$n_{77}$ & $-\cdots---$ & -- & $--1--10-$ \\
\hline$m_{78}$ & - & ------- & $-1--1-0-$ \\
\hline$m_{7}$ & $-\cdots$ & $-\cdots--0---$ & $----0-1-$ \\
\hline 1.08 & & & \\
\hline
\end{tabular}

\begin{tabular}{|c|c|c|c|}
\hline $\begin{array}{l}\text { chaining } \\
\text { variable }\end{array}$ & $31-24 \quad 23-16$ & $15-8$ & $8-0$ \\
\hline$a_{0}$ & 0110011101000101 & 00100011 & 00000001 \\
\hline$a_{1}$ & 010-FrF0 y0-01-0- & $10-0-10-$ & F-Fa0101 \\
\hline$a_{2}$ & F100-Vv1 0aa10a1a & 01a1a011 & 1-wa11a1 \\
\hline$\overline{a_{3}}$ & $01011 \mathrm{VFV}-1000000$ & 00000000 & 01FFa0a1 \\
\hline$\overline{a_{4}}$ & 0 w101v-a $\quad y--10000$ & 00101000 & $010 X W F 10$ \\
\hline$a_{5}$ & 0w0101y1 V1-11110 & $00111-00$ & 10010100 \\
\hline$a_{6}$ & 1w0a1a0a a0a1aaa- & $--10010 \mathrm{~F}$ & -W01F0Fh \\
\hline$a_{7}$ & wW0w0111 11111111 & $111-010 \mathrm{~F}$ & 0w0W0110 \\
\hline$a_{8}$ & w10wvv01 11110000 & $010-111 \mathrm{~F}$ & 1-Wh000F \\
\hline$a_{9}$ & 00WV--11 11111111 & $111----0$ & $---\mathrm{F} 1 \mathrm{~F} 01$ \\
\hline$a_{10}$ & W11x-Vvv --------- & $-----a--$ & -1 ww1h0w \\
\hline$a_{11}$ & 100V---- --------- & -------1 & $-1 \mathrm{hh} 0 \mathrm{hWw}$ \\
\hline$a_{12}$ & wwWF-v-- --------- & -------- & $-1 \mathrm{hhhhOh}$ \\
\hline$a_{13}$ & OwW--V-- -F-F-F-- & FNqNqqqq & q1hhh0WW \\
\hline$a_{14}$ & 1WWhhhhh hhhhhhhh & hNhNqNNq & NNhhh1wh \\
\hline$a_{15}$ & WWwhhhhh hhhhhhhh & hqhhqqqq & qNwh0hh0 \\
\hline$a_{16}$ & w1Whhhhh hhhhhhhh & hhNhqqqq & hqwh1hAh \\
\hline$a_{17}$ & $00-------\quad---------$ & --- & $----0-0-$ \\
\hline$a_{18}$ & $1-1-----\quad--------$ & ------- & $----a-0-$ \\
\hline$a_{19}$ & 0-b----- -------- & -------- & $\overline{----1}$ \\
\hline$a_{20}$ & --0----- -------- & -------- & $----a^{---}$ \\
\hline$a_{21}$ & --b----- -------- & -------- & $------0-$ \\
\hline$a_{22}$ & 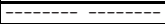 & ------- & $----a a^{--}$ \\
\hline$a_{23}$ & 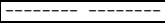 & -------- & ------00 \\
\hline$a_{24}$ & -c------- --------- & -------- & $----a$ \\
\hline$\overline{a_{25}}$ & -B------- --------- & -------- & $----a-0-$ \\
\hline$a_{26}$ & --------- -------- & --- & $----\mathrm{A} 1-$ \\
\hline$a_{27}$ & -------- -------- & --- & ---- \\
\hline$a_{28}$ & -C------- --------- & --- & $----a$ \\
\hline$a_{29}$ & -b------- -------- & -------- & $----a-1-$ \\
\hline$a_{30}$ & --------- -------- & ---- & $----\mathrm{A}_{0-}$ \\
\hline$a_{31}$ & 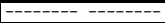 & --1 & $\overline{--}$ \\
\hline$a_{32}$ & - & -------- & --- \\
\hline$a_{33}$ & $-\mathrm{b}---------------$ & -------- & $----a-$ \\
\hline$a_{34}$ & -------- -------- & -------- & $---\mathrm{AAO}-$ \\
\hline$a_{35}$ & 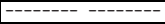 & 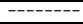 & ------00 \\
\hline$a_{36}$ & - c------- -------- & ------- & $----A---$ \\
\hline$a_{37}$ & -B------- ---------- & -------- & $----\mathrm{A}-1-$ \\
\hline$a_{38}$ & --------- --------- & -------- & $------0-$ \\
\hline$a_{39}$ & ---------- --------- & -------- & $\overline{------}$ \\
\hline$a_{40}$ & 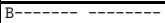 & -------- & $----A^{-}$ \\
\hline$a_{41}$ & $---------\quad---------$ & ---1 & -- \\
\hline$a_{42}$ & C------- -------- & -- & \\
\hline$a_{43}$ & B------- --------- & --------- & \\
\hline$a_{44}$ & 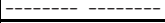 & --- & -- \\
\hline$a_{45}$ & $--------\quad--------$ & --- & --- \\
\hline$a_{46}$ & C-------- -------- & -- & -- \\
\hline$a_{47}$ & B-------- --------- & --- & \\
\hline$a_{48}$ & C-------- -------- & -------- & $----A_{-}^{-}$ \\
\hline$a_{49}$ & B-------- --------- & -------- & $------0-$ \\
\hline$a_{50}$ & C-------- --------- & --------- & $----A^{----}$ \\
\hline$a_{51}$ & B-------- --------- & -------- & $------0-$ \\
\hline$a_{52}$ & C-------- --------- & -------- & -- \\
\hline$a_{53}$ & B-------- --------- & -------- & $\overline{--}$ \\
\hline$a_{54}$ & --------- --------- & --- & \\
\hline$a_{55}$ & 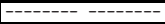 & --- & \\
\hline$a_{56}$ & --------- -------- & --1 & \\
\hline$a_{57}$ & 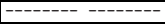 & --- & \\
\hline$a_{58}$ & $---------\quad--------$ & -------- & ---1 \\
\hline$a_{59}$ & ------------ & --1 & \\
\hline$a_{60}$ & $\overline{---------1}$ & -- & \\
\hline$a_{61}$ & --- & -- & \\
\hline$a_{62}$ & $\overline{-----------1}$ & -- & \\
\hline$a_{63}$ & ------ & $\overline{---}$ & -- \\
\hline$a_{64}$ & -- & -- & \\
\hline$a_{65}$ & 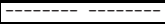 & --- & -- \\
\hline$a_{66}$ & --------- -------- & --- & $---\mathrm{A}--$ \\
\hline$a_{67}$ & --------- -------- & ---1 & -----0 \\
\hline$a_{68}$ & --------- --------- & --- & --- \\
\hline$a_{69}$ & --------- --------- & --------- & $--A----B$ \\
\hline$a_{70}$ & -------- --------- & -------- & $----0---$ \\
\hline$a_{71}$ & --------- -------- & -------- & $-----\mathrm{C}-$ \\
\hline$a_{72}$ & --------- --------- & -------- & $-A----B-$ \\
\hline$a_{73}$ & --------- -------- & --------- & $---0----$ \\
\hline$a_{74}$ & 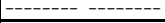 & --------- & $--\mathrm{A}--\mathrm{C}--$ \\
\hline$a_{75}$ & $--------\quad--------$ & -------- & $A---0 B--$ \\
\hline$a_{76}$ & --------- -------- & ---- & $--1---C-$ \\
\hline$a_{77}$ & |--------- & --------- & $----\mathrm{C}-\mathrm{B}-$ \\
\hline$a_{78}$ & ---------- --------- & --------- & $-----b---$ \\
\hline$a_{79}$ & ------- -------- & --------- & $-1-----$ \\
\hline$a_{80}$ & 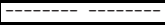 & & \\
\hline
\end{tabular}


For the full SHA-1, the CMM algorithm will be used to find a collision on 26round SHA-1. After the conventional message modification, it will remain the 9 following conditions; which are only related to rounds 17-26:

$a_{17,3}=0, a_{18,3}+a_{17,3}=0, a_{22,2}+a_{21,2}=0, a_{23,1}=0, a_{24,30}+a_{22,0}=0, a_{24,3}+a_{23,3}=0$, $a_{25,30}+a_{24,0}=1, a_{25,1}=0, a_{26,1}=1$.

Uncontrolled Relations. There is 64 uncontrolled relations on the $a_{i, j}$ :

$a_{27,0}=0, a_{28,30}+a_{26,0}=1, a_{28,3}+a_{27,3}=0, a_{29,30}+a_{28,0}=0, a_{29,3}+a_{28,3}=0$,
$a_{29,1}=1, a_{30,1}=0, a_{31,0}=1, a_{32,30}+a_{30,0}=0, a_{33,30}+a_{32,0}=0, a_{33,3}+a_{32,3}=0$,
$a_{34,3}+a_{33,3}=1, a_{34,2}+a_{33,2}=1, a_{34,1}=0, a_{35,1}=0, a_{35,0}=0, a_{36,30}+a_{34,0}=0$,
$a_{36,3}+a_{35,3}=1, a_{37,30}+a_{36,0}=1, a_{37,3}+a_{36,3}=1, a_{37,1}=1, a_{38,1}=0, a_{40,31}+a_{39,1}=$
$1, a_{40,3}+a_{39,3}=1, a_{41,1}=1, a_{42,31}+a_{40,1}=1, a_{43,31}+a_{42,1}=1, a_{44,3}+a_{43,3}=$
$1, a_{45,1}=0, a_{46,31}+a_{44,1}=1, a_{46,3}+a_{45,3}=1, a_{47,31}+a_{46,1}=1, a_{47,1}=0$,
$a_{48,31}+a_{46,1}=1, a_{48,3}+a_{47,3}=1, a_{49,31}+a_{48,1}=1, a_{49,1}=0, a_{50,31}+a_{48,1}=1$,
$a_{50,3}+a_{49,3}=1, a_{51,31}+a_{50,1}=1, a_{51,1}=0, a_{52,31}+a_{50,1}=1, a_{53,31}+a_{52,1}=1$,
$a_{66,4}+a_{65,4}=1, a_{67,2}=0, a_{68,0}+a_{66,2}=1, a_{69,5}+a_{68,5}=1, a_{69,0}+a_{68,2}=1$,
$a_{70,3}=0, a_{71,1}+a_{69,3}=1, a_{72,6}+a_{71,6}=1, a_{72,1}+a_{71,3}=1, a_{73,4}=0, a_{74,5}+a_{73,5}=1$,
$a_{74,2}+a_{72,4}=1, a_{75,7}+a_{74,7}=1, a_{75,3}=0, a_{75,2}+a_{74,4}=1, a_{76,5}=1, a_{76,1}+a_{74,3}=1$,
$a_{77,3}+a_{75,5}=1, a_{77,1}+a_{76,3}=1, a_{78,3}+a_{77,5}=0, a_{79,6}=1$.

To adjust these 64 conditions, we have tried to use semi-neutral bits and adjusters as explained in the IMM algorithm. We use 10 semi-neutral bits (corresponding to ' $N$ ' in Table 8) and 8 adjusters which are the bits 1-bit-left to ' $N$ '. We present an example of message $m=\left(m_{0}, m_{1}, \ldots, m_{15}\right)$ obtained by Algorithm 2

$m=b 8550 b b 25 b 2 e 1 a 1588 a 0 e 568$ b0d7cbaf $0 b 430105$ 1e7f1b5e $0637 d a 310 d c 9 d 562$ $7 d 857448$ defac00e $9 d 06 b a 9 e$ 2dd8235a 324e9acb f7c56578 c69dfd0e 71bf1d08

The above $m$ satisfies all message conditions of 0-80 rounds and all chaining variable conditions of $0-28$ rounds. 